Du texte à la scène : langages du théâtre

\title{
De Leir à Lear : mise en texte et mise en scène d'une légende et d'une politique
}

Jean Dubu

\section{(2) OpenEdition}

Journals

\section{Édition électronique}

URL : http://journals.openedition.org/shakespeare/459

DOI : 10.4000/shakespeare.459

ISSN : 2271-6424

Éditeur

Société Française Shakespeare

Édition imprimée

Date de publication : 1 novembre 1983

Pagination : 35-48

Référence électronique

Jean Dubu, « De Leir à Lear : mise en texte et mise en scène d'une légende et d'une politique », Actes des congrès de la Société française Shakespeare [En ligne], 4 | 1983, mis en ligne le 01 janvier 2007, consulté le 30 avril 2019. URL : http://journals.openedition.org/shakespeare/459 ; DOI : 10.4000/ shakespeare.459 
SOCIETE FRANÇAISE SHAKESPEARE

Actes du Congrès 1982

\section{DU TEXTE A LA SCENE : Langages du Théâtre}

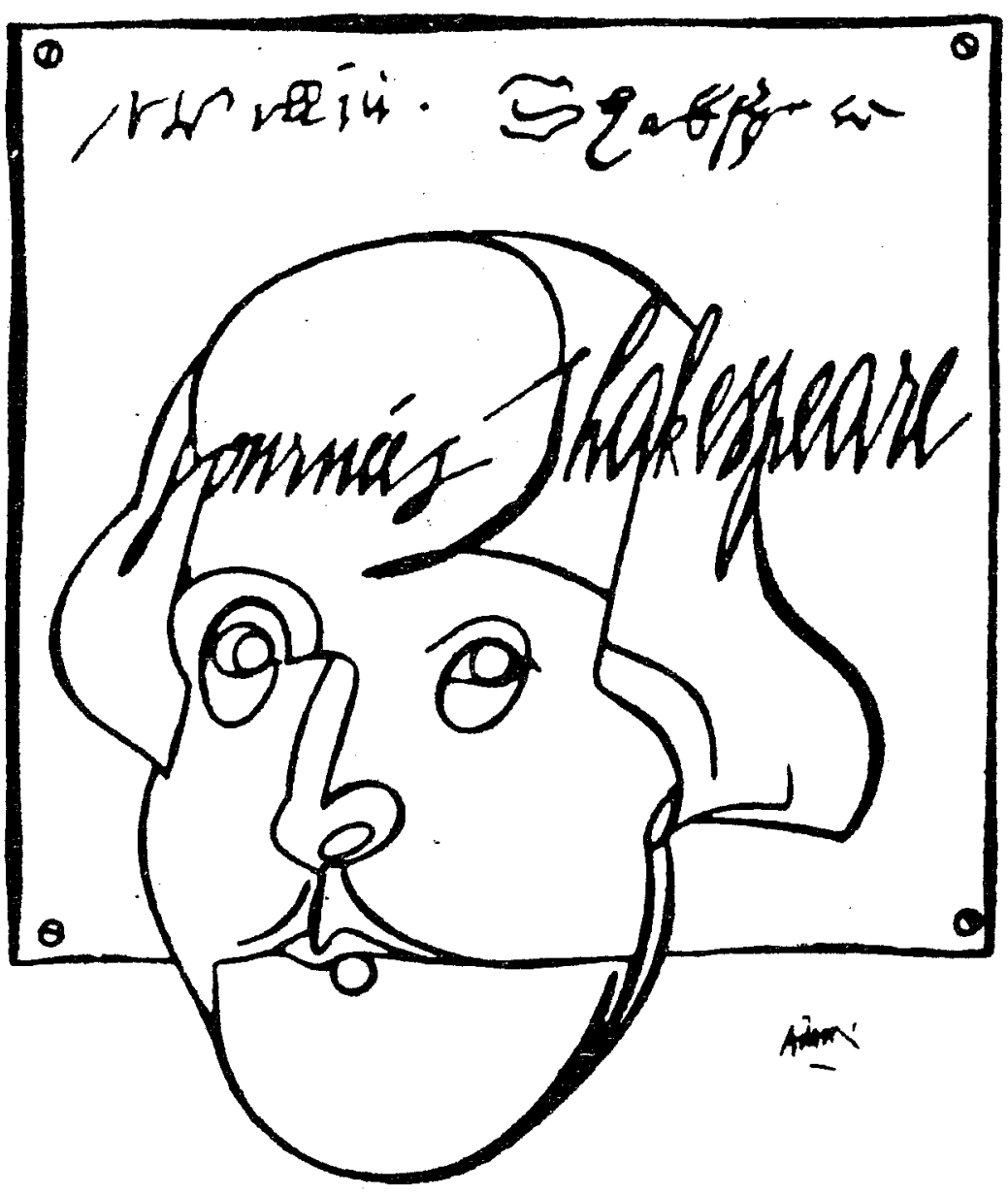

DiRecteur de la publication M.T. Jones - Davies

Publié avec le concours du Centre National de la Recherche Scientifique

JEAN TOUZOT Libraire - Editeur

38 , rue Saint-Sulpice 75278 PARIS CEDEX 061983 


\section{DE LEIR A LEAR: MISE EN TEXTE ET MISE EN SCENE D'UNE LEGENDE ET D'UNE POLITIQUE}

On ne saurait dire que la liste des rois de GrandeBretagne donnée par Edmund Spenser au chant $\mathrm{X}$ du second livre de The Faerie Queene brille par la clarté, même si elle fournit au spectateur et au lecteur de King Lear quelques aperçus intéressants sur la genèse et la signification de la pièce. De ces brumes épiques et légendaires, Shakespeare a tiré les quelques notions précises et simples utiles à l'exposition de sa tragédie. Remarquons en premier lieu chez Spenser et les anciens chroniqueurs britanniques un antécédent qui sert d'archétype. Nous apprenons (st. 13) que Locrine, fils aîné de Brute, devint à la mort de son père et par la volonté de celui-ci «chief Lord of Britany», et encore :

Locrine was left the soveraine Lord of all $(14,1)$ tandis que ses deux frères:

Albanact had all the Northrene part

Which of himself Albania he did call

et l'autre :

Camber did possesse the Westerne quart

Which Severn now from Logrie part.

$(14,4-5)$

Brute, en mourant, semble donc avoir instauré une hiérarchie des royaumes; c'est pourquoi, treize strophes plus loin nous acceptons que Leyr, héritier de Bledud, le fondateur de Bath, sans descendance mâle, marie ses trois filles à trois rois, dont deux au moins le sont en Grande-Bretagne : Goneril l'ainée épouse Maglan, roi des Ecossais, et Regan le roi de Cambrie; sans dot, Cordelia sera mariée à Aganip roi de Celtique. C'était le temps de la légende; Shakespeare écrit pour un public averti de l'histoire, l'esprit même ouvert à la politique, ne fût-ce que par les nombreuses controverses auxquelles la récente succession d'Angleterre avait donné un regain d'intérêt. Dans cette perspective, la liste des personnages de la tragédie, dramatis personae, 
ne laisse pas d'instruire.

$\mathrm{Si}$ l'orthographe de son nom, sur laquelle nous reviendrons, se trouve modifiée, Leir conserve son titre de «king of Britain»; mais de roi d'Ecosse, Maglan, l'époux de Goneril devient duc d'Albany, et de roi de Cambrie, l'époux de Regan devient duc de Cornouailles. Pour le premier de ces deux changements, il suffit de se reporter au second fils de Brute que nous avons cité; il a donné son nom à l'Albania, province dont Spenser fait la première retraite de Leir, une fois le royaume partagé:

The aged Syre, thus eased of his Crowne

A private life led in Albania.

$(29,6-7)$

Pour le second changement, de roi de Cambrie à duc de Cornouailles, on notera d'abord que Spenser a fourni antérieurement l'étymologie et l'origine de la Cornouailles:

Corineus had that province utmost West

To him assigned for his worthy lot,

Which of his name and memorable gest

He called Cornwaile, yet so called hest.

$(12,2-5)$

En second lieu, on rappellera qu'à l'époque où Shakespeare écrit, Albany et Cornwall sont devenus apanages princiers, dévolus respectivement par les deux souverains d'Ecosse et d'Angleterre à des membres de leur famille, et non des mémoires. Traditionnellement, le prince de Galles est duc de Cornouailles; quant au titre de duc d'Albany, Marie Stuart l'avait conféré à son second époux Darnley, il était passé à leur fils Jacques qui devait le transmettre à son second fils Charles. Dans le même ordre d'idées, on remarquera que Gloucester et Kent sont apanages royaux ici détenus par des membres subalternes de la hiérarchie nobiliaire; leur principal mérite, c'est leur notorité qui permet d'ancrer la légende dans le devenir national. Ces deux derniers personnages, d'ailleurs, sont des additions du dramaturge aux données de la légende. Par eux, la pyramide sociale se trouve confortée lorsque la pièce commence : au sommet, le roi, ensuite 
ses deux gendres titulaires d'un duché, le marì de l'aînée des filles en Ecosse, celui de la cadette en Angleterre de l'extrême ouest; les vassaux sont des Anglais du centre et du sud-est. La grande île est donc géographiquement représentée dans la tragédie, qui ne l'en concerne que davantage.

Dès la légende, Cordeill ou Cordelia était vouée à l'exil, si l'on doit assimiler la Celtique dont son mariage la fait reine à notre Bretagne, Gallia Celtica; respectueux jusqu'au scrupule des notions protocolaires, Shakespeare lui donne deux prétendants, l'un de rang égal à celui de son père, le roi de France, l'autre à celui de ses beauxfrères, mais Bourgogne, duc souverain, a la préséance sur les ducs britanniques. Il n'en partage pas moins avec eux la soif des biens de ce monde et une particulière cécité aux véritables valeurs morales. Ce sont là traits reconnus des grands feudataires, insatiables et peu délicats, thème cher aux théoriciens de l'absolutisme royal, au nombre desquels se rangeait Jacques Stuart. Ce duc avide n'épousera pas une princesse déshéritée qui répugne aux habiletés du monde; il s'en explique sans vergogne. Il ne faut pas moins que la clairvoyance d'un roi et la générosité chevaleresque d'un Français pour apprécier le caractère de Cordelia : en devenant reine de ce côté-ci de la Manche, elle occupera un rang tenu depuis par la reine-martyre, Marie Stuart, la propre mère du souverain, qui avait même demandé par testament d'être inhumée au pays de sa jeunesse heureuse ...

Si finalement elle repose à Westminster dans le tombeau que l'on sait, en symétrie avec sa cousine Elisabeth| n'est-ce pas précisément que leur commun successeur entend affirmer qu'il tient de l'une comme de l'autre l'un de ses sceptres et l'une de ses couronnes? Dès le début de son règne, il a tiré de ce point très particulier un programme politique, et il le signifie publiquement - non sans un certain éclat - en deux occasions au moins : celui qui par le jeu des successions est devenu le chef des deux Etats considère comme hautement sou- 
haitable que la réunion des deux titres royaux en sa personne soit le prélude à l'union des deux royaumes.

On peut le remarquer d'abord sur ses monnaies : la première série frappée à son arrivée à Londres se contentait d'insérer l'Ecosse dans la liste des Etats auxquels traditionnellement prétendait le roi d'Angleterre, il y est donc décrit comme Angliae, Scotiae, Franciae \& Hiberniae Rex. L'année suivante il devient : Britanniae, Franciae \& Hiberniae Rex. Britanniae Rex, King of Britain : c'est la titulature de Lear dans notre pièce, ce Lear dont une simple permutation de lettres comme on les goûte outreManche permet de transformer la nouvelle orthographe en real, ou réal, également significatifs du point de vue politique.

L'indispensable couronnement à Westminster Abbey, précédé d'un sacre avec onctions quasi-catholiques (depuis le sacre d'Henri VI à Notre-Dame de Paris, le rituel n'est-il pas le même que celui de Reims, calqué sur le sacre des évêques dans l'Eglise romaine ?), ce couronnement indispensable aux yeux de la majorité des Anglais présentait pour Jacques des risques politiques certains: celui de lui aliéner une fraction non négligeable de ses sujets nouveaux et anciens, d'humeur plus calvinisante, et de reléguer au second plan la couronne d'Ecosse, ceinte jadis avec moins de faste, et le risque de paraître oublieux de ses origines en se laissant angliciser, voire anglicaniser. Le souverain imagina donc de réaffirmer son programme d'union des deux couronnes, et ce dans le défilé qui le menait à Westminster, défilé dans lequel figurèrent les King's Men - et donc Shakespeare. Le souverain fit confectionner à grands frais, et porta sur son chapeau pour retenir son panache de plumes, un joyau extraordinaire nommé «Mirror of Great-Britain». L'inventaire des joyaux royaux de 1606 le décrit ainsi :

a greate and Riche Jewell of gould, called the Mirror of Greate Britaine, containing one very faire table diamonde, one very faire tablerubie, two other large diamonds cutt lozen- 
gewise, the one of them called the stone of the letter $\mathrm{H}$ of SCOTLANDE, garnished with small diamonds, two rounde pearles fixed, and one fayre diamonde cut in fourcetts, bought of Sancy. ${ }^{1}$

Ce dernier diamant, Jacques venait de l'acquérir aux frais de son nouveau royaume pour la somme considérable de 60.000 thalers; il avait appartenu à Charles le Téméraire, mais sa proche origine française pouvait lui permettre de symboliser notre pays à côté du diamant en losange, gravé d'un $\mathrm{H}$, provenant du trésor royal d'Ecosse et de pierreries du trésor royal d'Angleterre|En ce matin du 21 mars 1604, en un moment particulièrement solennel, c'était redire, symboliser le grand programme politique du règne; moins la vanité d'un prince devant sa promotion personnelle que l'accession pour chacun des deux royaumes, et par l'entremise de celui qui en était le vivant dépositaire, d'une puissance infiniment supérieure à la simple somme des deux, pour peu que ceux-ci acceptassent le principe de l'union, sinon de la fusion. C'était l'intérêt bien compris de tous que de le suivre, et non d'écouter ses détracteurs qui entendaient s'en tenir au statu quo, flattant ainsi les particularismes anglais et écossais. Outre la démonstration ostentatoire que constituaient monnaies et joyaux ${ }^{2}$, il semble bien que Jacques allait pouvoir compter pour conforter discrètement ses vues, au moins dans l'esprit de ses sujets cultivés et férus de théâtre, sur certains aspects de la tragédie encore à venir de son auteur dramatique favori.

Car sur le plan politique, le partage auquel procède Lear instaure justement l'état de division dont on sait les conséquences funestes : rivalités des principicules, combats, catastrophes. Tout cela est l'oeuvre d'un souverain âgé, si peu conscient de ses responsabilités qu'il songe à ses aises, au retour à la vie privée, et que, d'ailleurs, il perd il a sans doute déjà perdu - la raison. Si l'on mettait un instant entre parenthèses cette dimension proprement tragique, le péril d'Etat, nous nous trouverions devant 
un drame bourgeois, l'erreur d'un vieillard, citoyen privé, qui se dépouille de son bien pour doter des filles, avec toutes les conséquences personnelles et familiales qui découlent de dispositions prises à mauvais escient; nous débouchons sur le drame balzacien.

$\mathrm{Si}$, à l'opposé, nous songèons aux visées de Jacques Ier, le choix du sujet n'apparaît plus innocent, ni gratuit; il comporte une leçon politique a contrario : la division de la Grande-Bretagne en états distincts ne peut amener que des catastrophes. En songeant à y remédier dès son accession au trône d'Angleterre, en proposant de passer de l'union de fait en sa personne à l'union de droit, Jacques ne se révèle ni un utopiste, ni un mégalomane, voire un parvenu. Sans doute y avait-il de la distance, sur l'échiquier européen, du petit royaume très médiocrement peuplé d'Ecosse, et pauvre en ressources naturelles, et l'Angleterre en pleine expansion. Les buts du souverain n'en procèdent pas moins d'une intuition politique sage, fondée sur le précédent et les leçons de l'histoire (ou de la légende discrètement historicisée). La tragédie, école des princes, divertissement par excellence de la classe politique, peut le montrer. Depuis, notamment, la suppression de la scène de la déposition de son Richard II, Shakespeare ne peut ignorer la portée reconnue de son art dans ce domaine délicat. Le fou ne se gêne pas pour montrer à Lear la sottise de la politique de partage/division qu'il a appliquée:

F: Nuncle, give me an egg, and I'll give thee two crowns.

$\mathrm{K}$ : What two crowns shall they be ?

$\mathrm{N}$ : Why, after I have cut the egg i'th' middle and eat up the meat, the two crowns of the egg. When thou clovest thy crown i'th' middle and gavest away both parts, thou bor'st thine ass on thy back o'er the dirt. (I.iv. 155-159) La folie de Lear est donc moins un châtiment que la preuve de son aveuglement tragique, son erreur, On notera à ce sujet que, ni chez Spenser, ni chez les chroniqueurs, 
Lear n'est désigné comme fou, mais seulement comme l'auteur d'une erreur humaine et politique. Le dramaturge voit bien toutes les ressources que lui offre le spectacle de la folie pour faire passer la leçon.

L'erreur initiale du vieux roi en entraîne logiquement d'autres. De sang royal, Goneril et Regan se trouvent mariées au-dessous de leur rang; mais cette circonstance voulue par l'auteur - tout comme la seconde intrigue empruntée à l'Arcadia - permettent de mettre au grand jour l'absence de sens politique des deux femmes. Comme leur père, elles ont tendance à faire passer leurs aspirations propres, leurs pulsions intimes, désirs et convoitises, avant le respect des engagements pris.

A la perversion qui a consisté pour le roi à rechercher ses aises et à démembrer son royaume, à la sottise qui a consisté pour le père à se mettre à la merci de ses filles, Goneril et Regan viennent ajouter le mépris pour leur mari respectif, leur infidélité conjugale, leur préférence pour un bâtard et, sans ambages, leur simple satisfaction physique. Face à ces deux femmes dénaturées et à ce vieillard, Shakespeare dispose un échantillonnage de la noblesse britannique; il est clair qu'il faut descendre Cordelia exceptée - aux comtes et gentilshommes pour rencontrer des exemples accomplis d'humanité, des personnages courtois, hospitaliers, compatissants, mesurés, discrets jusque dans le sacrifice, alliant la douceur chrétienne à la résignation stoïcienne. Dès la première scène Kent fait preuve de clairvoyance, et se voit confier par l'auteur une véritable mission prophétique - chargée d'ironie tragique - que l'aveuglement des autres protagonistes ne leur permet pas de comprendre. A ces autres, aux ducs, surtout Cornwall le mutilateur, tous acoquinés avec Edmund le Bâtard, Shakespeare réserve les comparaisons avec les animaux les plus sauvages et les plus nuisibles. Albany est relativement épargné : son nom, peut-être, le préserve d'un excès d'ignominie ...

Cordelia se situe sur un tout autre registre qui, mais est-ce absolument fortuit ?... nous ramène vers Jacques Ier 
et les joyaux. Selon la chronique, Cordelia parvint a rétablir son père sur son trône, où il acheva paisiblement ses jours; elle lui succèda et régna sagement, mais la rébellion victorieuse de ses neveux la conduisit en prison où elle se pendit. Bien des raison militaient pour que Shakespeare modifie quelques-unes de ces données : ne lui fallait-il pas en premier lieu resserrer l'action dans le temps ? C'est la loi du genre tragique, par opposition à l'épopée. Maniant une fois de plus l'ironie dramatique, il frappe Goheril et Regan de la stérilité dont Lear nouvel exemple de sa déraison - avait menacé l'aînée. Ceci ne veut pas dire que, faute de neveux rebelles, Corcielia sera sauve; elle mourra quand même en prison, mais - autre point commun avec la mère de Jacques, et qui contribue à la qualité «royale» de l'image que nous devons en avoir, princesse, reine consciente de ce qu'elle se doit à elle-même, il lui épargne le désespoir. Parachevant son régicide, Edmund lui sera aussi fatal qu'à ses soeurs : la trahison du bâtard épargne à la reine de France la tentation/trahison, felo de se. Dans l'optique chrétienne qui est celle de Shakespeare, le suicide est la mort des traîtres.

Le retour sur scène de la jeune femme ne se produit qu'à la scène iv de l'acte IV, mais juste avant, un récit nous informe de sa première rencontre avec son père; nous y entendons une description minutieuse de l'attitude de la jeune femme lorsqu'elle a appris le sort cruel reservé au vieux monarque. Le gentilhomme qui parle sait son monde et la Cour; n'a-t-il pas suivi la princesse en France? Sur les lèvres de cet anonyme vient se placer la plus riche, la plus royalement évocatrice de toutes les métaphores de la pièce :

...patience and sorrow strove Who should express her goodliest. You have seen Sunshine and rain at once; her smiles and tears Were like, a better way; those happy smilets That played on her ripe lip seemed not to know What guests were in her eyes, which parted 
thence

As pearls from diamonds dropped.

(IV. iii. 17-23)

As pearls from diamonds dropped : une curieuse médaille $\mathrm{du}$ début du règne qui commémore la paix de 1604 avec l'Espagne, montre Jacques de face, portant un chapeau à panache surmonté d'une couronne royale, d'un effet, il faut le dire, peu banal; le plus important pour nous c'est que sous le rebord du chapeau apparait un autre joyau, un diamant carré en table appelé - la coïncidence vaut d'être notée - «The Mirrour of France», qui surmonte une perle poire laquelle semble littéralement en dégoutter. On retrouve d'ailleurs le même joyau porté par la reine Anne, épouse de Jacques ${ }^{3}$, et les deux documents nous permettent de juger du réalisme du poète. Si de pareils joyaux signalent les souverains, le poète dont le verbe est la seule richesse et l'unique ressource a su réserver pour caractériser l'unique personnage de sa pièce qui soit digne du trône, l'image que ce bijou pouvait lui inspirer, laquelle, de ce fait, devient emblématique. On peut même remarquer que cette image a été ironiquement annoncée dès l'acte I, où la première parole de Cordelia après le départ de son père furieux contre elle, parole accompagnée d'un geste de dépouillement volontaire, c'est :

The jewels of our father, with washed eyes, Cordelia leaves you.

(I. i. 268-269)

L'association dans les deux cas des larmes et des bijoux, ainsi que la rareté dans toute l'oeuvre de ce type d'image ajoute à la signification. Lelcontraste aussi : souvenonsnous des tigresses, louves, renardes et autres ourses dont l'auteur use à l'égard des soeurs aînées. L'unique allusion qu'il se permette encore à des pierres précieuses dans tout le reste de la pièce, se trouve d'ailleurs sur les lèvres d'Edgar, ce double, par certains côtés, christique de Cordelia et futur roi : voici comme le jeune homme dépeint les yeux perdus de Gloucester :

$\ldots$ in this habit 
Met I my father with his bleeding rings, Their precious stones new lost.

(V.iii. 187-189)

La métonymie est d'autant plus parlante que l'usage de la dénotation, précious stones, fait passer de l'horreur (bleeding) à l'idée toute négative de cécité : cependant le registre est royal, il sied parfaitement à un jeune gentilhomme appelé quelques moments plus tard à ceindre la couronne.

On n'évoquera que d'un mot le contresens de Nahum Tate ${ }^{4}$ lorsqu'il entreprit de travestir Shakespeare et de donner à notre pièce un dénoûment heureux, fidèle certes à la légende, mais incompatible avec le genre tragique adopté par l'auteur. Un critique français du XVIle siècle nous paraît s'être fort heureusement exprimé sur ce point :

... L'une des plus grandes beautés que le poète puisse former dans la structure de sa fable, c'est de faire que l'aventure qui doit finir tragiquement aille bien avant dans la joie, avant d'être troublée par les accidents funestes qui composent la catastrophe ...

ce qui justifie pour une bonne part l'accalmie du retour et même de l'arrestation de Lear :

We two alone will sing like birds i'th'cage

(V. iii. 9)

Quel destin, au moins pour une jeune femme qui aurait pu rester libre, et reine de France !... La comparaison montre la limite de la reconnaissance de l'erreur, de l' anagnorisis qui s'est produite en lui; la raison n'est guère revenue, qui prend pour idéal le sort de l'oiseau en cage : nouvelle et discrète allusion aux dix-neuf dernières années de Marie Stuart ? A ce degré d'inconscience, peut-on sérieusement envisager qu'il exerce encore le pouvoir royal ? Evidence psychologique et nécessité politique concordent avec l'impératif tragique. Sur un point précis, les épreuves subies ont été perçues exclusivement comme les conséquences de l'«ingratitude»; c'est demeurer au plan de la causalité 
psychologique :

A very foolish fond old man More sinned against than sinning

$$
\text { (IV.vii. } 60 \text { et III. ii. 60) }
$$

Voilà sans doute de quoi exciter notre pitié selon la définition d'Aristote; mais il existe un péché des rois, qui est de refuser de régner, et de ce péché-là Lear n'apparaît à aucun moment convaincu ou conscient. Il est au moins autant qu'Edmund responsable à longue échéance de la mort de Cordelia. Témoins du partage initial, de ses raisons pompeusement établies, nous en avons vu les fâcheuses conséquences se développer inexorablement et nous ne pouvons ignorer sa culpabilité. Mais remonter à son erreur personnelle initiale l'amènerait à prendre conscience d'une vérité que le clairvoyant Gloucester pour sa part n'a jamais oubliée. Pour le fidèle vassal, en la personne du roi, il convient d'épargner

« his anointed flesh»

(III, vii. 57)

L'onction de Westminster, à la différence du serment d'Edimbourg vaut l'onction sacerdotale : Tu es rex in aeternum. Il ne saurait y avoir d'interruption de la fonction royale qu'à la mort de l'intéressé, et nulle possibilité d'intermittence. Cordelia aura beau user d'une manière de psycho-thérapie en entourant son père des égards royaux, en le faisant revêtir de manière convenable :

Enter Lear, asleep in a chair carried by servants, [clad in his royal robes.]

(IV. vii. 24)

toute cette mise en scène reste dérisoire devant l'évidence tragique, qui est la même évidence politique dont meurt Richard II. Sur le plan strictement social, la réussite de Cordelia est patente, et fallacieuse : la politesse de cour (amèrement dénoncée par Lear dans le monologue «We two will sing...») reprend le dessus; à la fin, même désastreuse, de la lutte, tous, Albany compris, parlent du Roi en se référant à Lear. Mais la jeune femme s'abuse si elle croit qu'il puisse ne s'agir de rien autre que du respect 
des convenances. C'est la raison pour laquelle elle doit aussi mourir; elle n'a pas davantage compris qu'on ne remonte pas impunément sur le trône après l'avoir volontairement abandonné, et pire encore après avoir démembré le royaume. Albany ne montre pas plus de clairvoyance : ne propose-t-il pas, non sans beaucoup de naïveté, de reprendre les errements qui ont précisément mené à la catastrophe ? Kent le comprend : partager le pouvoir, avec Edgar ou quiconque, est impossible. La discrétion qui sied à son état de mourant, et son tact inné, le conduisent à refuser l'offre d'Albany :

Rule in this realm, and the gored state sustain. (V.iii. 319) L'ambiguité du terme «gored» dit le poids de l'aveuglement de Lear et ses conséquences désastreuses. En choisissant pour sujet de sa première tragédie écrite depuis l'avènement de son protecteur de porter à la scène un roi dément qui démembre son royaume, Shakespeare soulignait avec tact mais sans équivoque possible, la sagesse politique du grand dessein d'union de la Grande-Bretagne cher à Jacques Ier. C'était se montrer à la fois digne et reconnaissant de la protection dont sa troupe et lui-même avaient été l'objet. 
1. Voir le Catalogue de l'exposition Princely'Magnificence, Court Jewels of the Renaissance 1500-1630 (15 oct. $1980-1$ fév. 1981) Londres, Victoria \& Albert Museum, 140 p. $24 \times 27$ avec ill. en couleurs. p. 107 (cote P. 19)

2. On remarquera que sur le portrait d'apparat maintenant à la National Portrait Gallery, peint par Daniel Mytens, à côté du souverain assis «in $h$ is conoration robes» et tete nue, figure sur une table, non une couronne (il aurait fallu choisir...), mais le chapeau au panache de plumes blanches retenu par le joyau déjà décrit.

3. Catalogue de l'exposition cité n. 1, p. 108 , cote P. 21.

4. Voir à ce sujet la communication de Mme Willems : «Du texte shakespearien à la scène de la Restauration et du XVIIle siècle.»prononcée au cours du même congrès.

5. Saint-Ussans, Réponse à la critique de la Bérénice de Racine, 1671 . 